\section{Remove the guesswork to better protect patients}

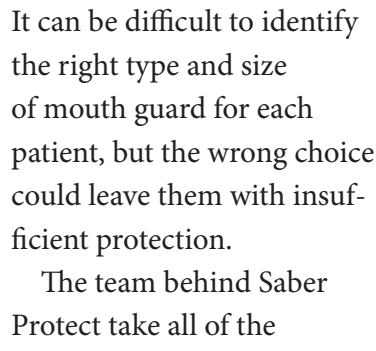

The specialist team of dental technicians will create a mouth guard to fit precisely within your patient's mouth. They will also customise the design to meet specific requirements, with the multi-layered material built up more for high contact or professional level sports and vice versa.

All you have to do is take an impression of the patient's mouth, fill out a form and send both to the laboratory - within ten days a one-of-a-kind Saber Protect mouth guard will be back in your practice ready for your patient.

For a mouth guard that is leaps and bounds ahead of others in the industry, and one that will protect your patients' future oral health and facial appearance, choose Saber Protect.

Saber Protect mouth guards are fabricated by CosTech Elite. For more information, visit www.custom-mouth-guards.com.

\section{Game-changing restorative solutions}

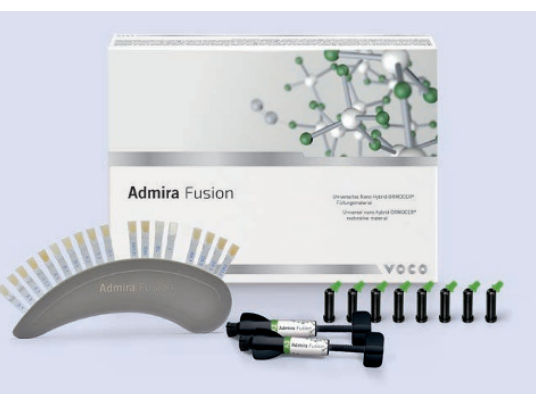

From fundamental concepts to advanced skills, let VOCO take you on a restorative dental materials journey that focuses on physical properties, biocompatibility, aesthetics and ease of application.

VOCO have used the latest advances in science, combining proven nanohybrid and

ORMOCER technology ('Organically Modified Ceramics') to develop Admira Fusion which offers several remarkable clinical advantages. As the world's first dental restorative material to use this unique 'Pure Silicate Technology', the light-curing and radiopaque Admira Fusion delivers a highly biocompatible material for anterior and posterior use. With $84 \%$ (by weight) content of inorganic fillers which delivers outstanding handling in comparison with all restorative composites of market relevance, it exhibits by far the lowest level of polymerisation shrinkage ( $1.25 \%$ by volume) coupled with extremely low shrinkage stress.

Offering excellent handling and high surface hardness to guarantee first-class long-term results, Admira Fusion has the optimal selection of shades for single or multiple shade techniques and can be used with all conventional bonding materials.

Change your game with VOCO - Trial Admira Fusion by requesting a free 'Starter Kit' when you order your first treatment kit. Call the expert VOCO team today free on 0080044444555 or email service@voco.de.

www.voco.com

\section{Ideal solutions for wet and dry milling}

Visitors to the Roland DG stand at the upcoming International Dental Show (IDS) in Cologne (Hall 3.1 Booth L030/M029) are invited to discover the exciting clinical and commercial benefits of CAD/CAM in modern day dentistry and discover why so many dental professionals worldwide choose Roland DG milling machines as part of their open system digital dental workflow.

In addition to showcasing some exciting product innovations in its range of market-leading technology, Roland DG will be demonstrating the popular DWX51-D 5-axis dental milling machine, highlighting its reliability and ability to dry mill popular composite resins, zirconia, PEEK and other materials.

Also on display will be the DWX-4W

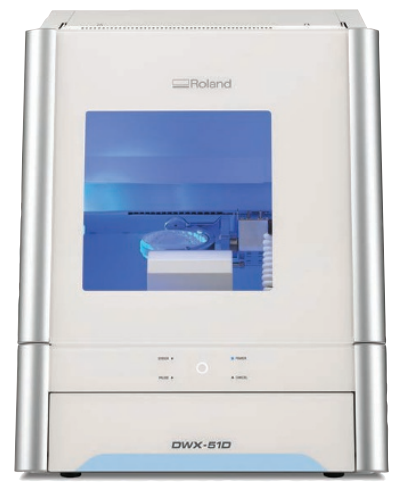
dedicated wet mill, ideal for fast, precision cutting of glass ceramics and composite resins, producing crowns, bridges, inlays, onlays, and veneers at high-speed thanks to its Jaeger DentaDrive spindle and simultaneous 4-axis milling.

With over 30 years' experience in manufacturing desktop milling technology, Roland DG has developed the ideal solutions for wet and dry milling, to deliver maximum productivity and avoid unnecessary drying and cleaning cycles.

With a comprehensive range of samples on the booth, visitors will discover the versatility and profit-making opportunities which are possible for today's dental professionals harnessing the potential of digital production.

http://rolandids2017.com/en/

\section{Follow nature's contour}

OsseoSpeed Profile EV from Dentsply Sirona is a unique implant specifically designed to follow the existing bone in sloped ridge situations, maintain soft tissue aesthetics and help reduce the need for bone augmentation.

It's well-known that crestal bone resorbs after tooth extraction or loss, and is often more pronounced on the buccal side, resulting in a lingual-to-buccal sloped ridge. This can occur even if a standard implant is placed immediately in the extraction socket. Bone-to-implant support is three-dimensional, making it important to have $360^{\circ}$ marginal bone support around the entire implant. When standard implants are placed level with the lingual bone, the implant neck can be exposed buccally, compromising the aesthetics of the final restoration. Implants placed level with the buccal bone on the other hand means unsupported lingual marginal bone is lost.

OsseoSpeed Profile EV is placed level with both buccal and lingual marginal bone, preserving soft tissue aesthetics and helping reduce the need for bone augmentation. This unique one-position-only placement makes the entire treatment procedure simple and predictable, from implant placement to connection of the final abutment.

Call 01932838346 or visit dentsply.com/en-uk. 